\title{
Sinopse das espécies de Panicum L. subg. Panicum (Poaceae, Paniceae) ocorrentes no Brasil ${ }^{1}$
}

\author{
Adriana Guglieri ${ }^{3,4}$, Fernando O. Zuloaga² e Hilda Maria Longhi-Wagner ${ }^{3}$
}

Recebido em 04/04/2003. Aceito em 13/10/2003

\begin{abstract}
RESUMO - (Sinopse das espécies de Panicum L. subg. Panicum (Poaceae, Paniceae) ocorrentes no Brasil). O levantamento de Panicum subg. Panicum revelou a ocorrência de 29 espécies para o Brasil. O trabalho apresenta chaves para diferenciar o subgênero Panicum de outras seções de Panicum atualmente não agrupadas em subgêneros definidos, e para espécies do subgênero Panicum, ilustrações de caracteres taxonômicos selecionados e dados de distribuição geográfica das espécies tratadas.
\end{abstract}

Palavras-chave: Gramineae, Panicum, subgênero Panicum, Brasil

\begin{abstract}
Synopsis of Panicum subg. Panicum (Poaceae, Paniceae) in Brazil). A survey of Panicum subg. Panicum confirmed the occurrence of 29 species in Brazil. Analytical keys to differentiate the subgenus Panicum from other sections of Panicum not included in defined subgenera, and for species of subgenus Panicum are provided, as well as illustrations of taxonomic characteristics and data on their geographic distribution.
\end{abstract}

Key words: Gramineae, Panicum, subgenus Panicum, Brazil

\section{Introdução}

O gênero Panicum L. é um dos maiores e mais importantes da família Poaceae. De acordo com a circunscrição anteriormente aceita para o gênero, 470 espécies foram reconhecidas para o mesmo por Clayton \& Renvoize (1986) e aproximadamente 370 por Watson \& Dallwitz (1992).

Estudos mais detalhados com a utilização de diferentes abordagens têm levado ao desmembramento do gênero Panicum, aceitando a elevação de alguns subgêneros anteriormente propostos a categorias de gêneros, tais como Steinchisma Raf. (Zuloaga et al. 1998) e Dichanthelium (Hitchc. \& Chase) Gould (Gould 1974). O status de gênero para estes dois últimos foi confirmado por Aliscioni et al. (2003), que consideraram o subgênero Phanopyrum (Raf.) Pilg. como gênero independente, com apenas uma espécie da América do Norte. Das demais espécies anteriormente aceitas em Phanopyrum, parte foi transferida para outros gêneros da tribo Paniceae e parte permaneceu agrupada nas mesmas seções aceitas anteriormente, porém sem subgênero definido. O subgênero Panicum foi reconhecido como monofilético, com as seções Dichotomiflora (Hitchc.) Honda, Panicum, Rudgeana (Hitchc.) Zuloaga, Urvilleana (Hitchc.) Pilg. e Virgata Hitchc. \& Chase ex Pilg. Outras espécies aceitas anteriormente em Panicum foram mantidas no gênero, porém agrupadas apenas em seções, ainda sem subgêneros definidos (Aliscioni et al. 2003).

De acordo com a circunscrição atual (Aliscioni et al. 2003), o gênero Panicum "sensu lato", compreende cerca de 400 espécies, das quais cerca de 100 incluídas no subgênero Panicum, distribuídas em regiões pantropicais, algumas das quais se estendem até as regiões temperadas.

Nos últimos anos foram publicados vários trabalhos que tratam da revisão de alguns subgêneros e seções de Panicum, tais como Zuloaga (1987a; b; 1989; 1994), Zuloaga \& Morrone (1992; 1996a; b) Zuloaga et al. (1992a; b; 1993a; b) entre outros. Porém, o gênero Panicum no Brasil foi pouco estudado e a revisão do mesmo tem sido feita em algumas floras regionais.

Segundo Smith et al. (1982), 49 espécies de Panicum ocorrem no Estado de Santa Catarina. Destas, 10 espécies representam o subgênero Panicum e as seções Dichotomiflora (três espécies), Panicum

\footnotetext{
Parte da Tese de Doutorado da primeira Autora

2 Instituto de Botánica Darwinion, San Isidro, Argentina (fzuloaga@ darwin.edu.ar)

3 Departamento Botânica UFRGS, Porto Alegre, RS, Brasil. Bolsa CNPq (hmlw@ vant.com.br)

4 Autor para correspondência: aguglieri@bol.com.br
} 
(duas), Virgata (duas), Rudgeana (uma), Urvilleana (uma) e uma espécie agrupada a este subgênero sem seção definida.

Guglieri \& Longhi-Wagner (2000) citaram 36 espécies para o Estado do Rio Grande do Sul. Destas, 13 fazem parte do subgênero Panicum, com cinco representantes da seção Dichotomiflora, quatro de Panicum, duas de Virgata, uma de Urvilleana e uma espécie sem seção definida.

Zuloaga et al. (2001) constataram a ocorrência de 42 espécies de Panicum para o Estado de São Paulo, sendo quatro espécies da seção Dichotomiflora, três de Rudgeana, duas de Panicum, uma de Urvilleana, e uma espécie sem seção definida, totalizando 11 espécies do subgênero Panicum.

As espécies do subgênero Panicum distribuem-se desde o Canadá até a Argentina e o Brasil, com ocorrência confirmada em alguns países da Europa e Ásia, e por toda África e Oceania (Häfliger \& Scholz 1980). O referido subgênero está bem representado em todas as regiões brasileiras. Suas espécies habitam geralmente locais alterados, em ambientes variados como campos secos e úmidos, banhados e margens de arroios e rios. Este subgênero também se destaca por apresentar espécies de interesse econômico, como P. miliaceum L., o "painço", cultivada como alimento para aves, e P. racemosum Spreng., o "capim-dasdunas", com grande importância ecológica, atuando como fixadora de dunas primárias. Além disto, inclui espécies invasoras de áreas de cultivo e jardins, como $P$. aquaticum Poir., P. gouinii E. Fourn., $P$. dichotomiflorum Michx. e P. repens L.

O objetivo deste trabalho é o de fornecer meios para a identificação das espécies do subgênero Panicum ocorrentes no Brasil. Além disto, são fornecidos dados sobre a distribuição geográfica das espécies tratadas.

\section{Material e métodos}

Este trabalho foi baseado em revisão bibliográfica e de herbários, coletas e observação de populações no campo, além de estudos morfológicos detalhados.

Foram revisados os seguintes herbários, citados pela sua sigla internacional, conforme Holmgren et al. (1990): B, BA, BAA, BAB, BAF, BHCB, BLA, CEN, CEPEC, CNPO, COL, COR, CORD, CTES, CVRD, EAC, ESA, ESAL, F, FUEL, G, GH, HAMAB, HAS, HCB, HERBACRUZ, HEPH, HRCB, HST, HUCS, HUEFS, HUFU, HUI, HUM, HURG, HUPF, IAC, IAN, IBGE, ICN, INPA, INTA, ISC, JEPS, JPB, K,
LA, LE, LIL, LP, LPB, M, MAC, MBM, MBML, MEXU, MO, MPUC, MY, NY, P, PACA, PAMG, PEL, PMSP, PORT, R, RB, RFA, RSPF, S, SI, SMDB, SP, SPF, SPSF, TEPB, UB, UFG, UFMT, UPCB, US, USM, UTME, VEN, W e WIS. Além destes, foi revisado o herbário Balduíno Rambo, do Museu Regional do Alto Uruguai (Universidade Regional Integrada do Alto Uruguai e das Missões, URI), Erexim, RS, cuja sigla, não oficial, é HERBARA.

Os dados de distribuição geográfica foram retirados da literatura e do material examinado. Os dados de ambiente foram baseados nas informações contidas nas etiquetas de herbário e em observações feitas diretamente no campo. A maior parte das espécies foi observada e coletada em seu ambiente natural. Os caracteres de maior importância taxonômica foram ilustrados.

Está sendo citado apenas um exemplar selecionado de cada táxon, como testemunho, porém listagem completa pode ser solicitada à primeira autora.

\section{Resultados e discussão}

O gênero Panicum apresenta espiguetas com duas glumas caducas, dois antécios (raramente três), o inferior membranoso, neutro ou masculino, e o superior coriáceo, com flor bissexuada, caracteres gerais da tribo Paniceae. Caracteriza-se pela gluma inferior menor do que os antécios, espiguetas em panícula laxa a contraída, ou dispostas em ramos unilaterais. Panicum subg. Panicum é caracterizado pela ausência de quilha nas folhas, gluma inferior (5)7-11(13-15)nervada, panícula laxa a contraída, e metabolismo fotossintético $\mathrm{C} 4$, do tipo NAD-ME.

No Brasil ocorrem cerca de 114 espécies do gênero Panicum sensu lato, das quais 29 incluídas no subgênero Panicum, conforme dados do presente trabalho, e as demais distribuídas nas seguintes seções: Prionitia Zuloaga, Tenera (Hitchc. \& Chase) Pilg., Valida Zuloaga \& Morrone e Discrepantia Zuloaga, além de $P$. antidotale Retz. (grupo 1 na chave a seguir); Laxa Hitchc. \& Chase ex Pilg. p.p., Lorea Zuloaga, Megista Pilg., Monticola Stapf, Parvifolia Hitchc. \& Chase ex Pilg., Parviglumia Hitchc. \& Chase ex Pilg., e Stolonifera Hitchc. \& Chase ex Pilg. (grupo 2). As seções do primeiro grupo eram incluídas no subgênero Agrostoides (Hitchc.) Zuloaga, enquanto que as do segundo grupo no subgênero Phanopyrum, anteriormente ao trabalho de Aliscioni et al. (2003), que as mantiveram "incertae sedis", sem subgênero definido. 
Chave para diferenciar o subgênero Panicum das seções "incertae sedis" de Panicum ocorrentes no Brasil

1. Gluma superior (5-)7-11(13-15)-nervada. Panícula com as espiguetas dispostas irregularmente ao longo de todos os ramos subg. Panicum

1. Gluma superior 3-5-nervada. Panícula com as espiguetas dispostas irregularmente ao longo de todos os ramos ou dispostas em ramos primários unilaterais. Se glumas com número maior de nervuras, sempre espiguetas dispostas em ramos primários unilaterais.

2. Colmos maciços. Lâminas acanaladas, quilhadas, com margens fortemente escabras, cortantes. Panícula com as espiguetas dispostas irregularmente ao longo de todos os ramos grupo 1

2. Colmos ocos. Lâminas planas, com margens levemente escabras, não cortantes. Panícula geralmente com espiguetas dispostas em ramos primários unilaterais, menos comumente dispostas irregularmente ao longo de todos os ramos grupo 2

Chave para as espécies de Panicum subg. Panicum ocorrentes no Brasil

1. Espiguetas com três antécios, o primeiro neutro e membranoso, o segundo com flor estaminada e também membranoso, e o terceiro com flor perfeita e coriáceo 24. P. quadriglume

1. Espiguetas com dois antécios, o primeiro neutro ou com flor estaminada, membranoso, e o segundo com flor perfeita e coriáceo

2. Segundo antécio com indumento; espiguetas glabras ou vilosas, tricomas 1-5mm compr.

3. Segundo antécio com tricomas longos nas margens do lema; espiguetas em geral densamente vilosas; plantas com rizomas 25. $P$. racemosum

3. Segundo antécio com tufo de tricomas longos na base do lema, lateralmente; espiguetas glabras; plantas sem rizomas

4. Panícula apenas com ramificações de primeira ordem; segundo antécio com estípite

20. P. mystasipum

4. Panícula com ramificações de primeira, segunda e terceira ordens; segundo antécio sem estípite

21. P. olyroides

2. Segundo antécio glabro; espiguetas glabras ou pilosas, se pilosas, tricomas $0,2-0,6 \mathrm{~mm}$ compr.

5. Gluma inferior separada da gluma superior por um entrenó conspícuo, a superior inserida notadamente acima da inferior

6. Panícula (3-)4,5-6,5cm×(1-)1,5-3cm; segundo antécio sem estípite e com um anel circular castanho na zona de inserção com a ráquila

17. P. magnispicula

6. Panícula 11-45(-62)cm×(2-)5,5-27(-44)cm; segundo antécio com ou sem estípite e sem anel circular castanho na zona de inserção com a ráquila

7. Espiguetas 2,1-4,2(-4,4)mm compr.; segundo antécio sem estípite ou com estípite de até $0,6 \mathrm{~mm}$ compr.

8. Espiguetas glabras; plantas com ou sem rizomas

9. Plantas sem rizomas; espiguetas obovais

6. $P$. cayennense

9. Plantas com rizomas; espiguetas lanceoladas a oval-lanceoladas, ou ovais

10. Pedicelos escabros, sem tricomas longos; espiguetas 4-4,2(-4,4)mm compr; glumas superior e inferior com o ápice retorcido. Norte do Brasil (Maranhão)

1. P. altum

10. Pedicelos escabros ou não, geralmente com um a vários tricomas longos; espiguetas 2,2-3,4mm compr.; glumas superior e inferior com o ápice não retorcido. Centro e Sul do Brasil

11. Lâminas foliares glabras; espiguetas 2,2-2,6mm compr. 
11. Lâminas foliares com face abaxial glabra e face adaxial pilosa a hirsuta, tricomas esparsos a densos; espiguetas 2,4-3,4mm compr.

13. P. glabripes

8. Espiguetas esparsamente pilosas a hirsutas; plantas com rizomas

12. Espiguetas (2,9-)3,2-3,4mm compr., esparsamente hirsutas 27. P. rudgei

12. Espiguetas 2,5-2,9(-3)mm compr., esparsamente pilosas 5. $P$. campestre

7. Espiguetas 4,5-9mm compr.; segundo antécio com estípite de $1 \mathrm{~mm}$ compr. ou mais

13. Espiguetas 4,5-5,4mm compr.; estípite com base glabra; bainhas foliares hirsutas ou híspidas; lígula (1,5-)2,5-3,5(-5)mm compr. 16. P. ligulare

13. Espiguetas $6,9-9 \mathrm{~mm}$ compr.; estípite com base pilosa, com poucos tricomas esparsos; bainhas foliares glabras, pilosas ou hirsutas; lígula 0,6-1,5(-2,6)mm compr.

7. P. cervicatum

5. Gluma inferior separada da superior por um entrenó muito curto, inconspícuo, aparentemente inseridas no mesmo ponto

14. Espiguetas com a gluma inferior 1/6-1/3 do comprimento da espigueta, excepcionalmente até $1 / 2$ ou $3 / 4$ do comprimento; plantas decumbentes, com colmos radicantes nos nós inferiores ou eretas, de ambientes aquáticos ou próximos a cursos d'água

15. Espiguetas estreito-lanceoladas, (3,7-)4,6-5,4mm compr.; colmos suculentos com aerênquima

10. P. elephantipes

15. Espiguetas lanceoladas a oval-lanceoladas, (1,9-)2,4-3,4mm compr.; colmos sem aerênquima

16. Plantas eretas

17. Gluma inferior de ápice agudo ou obtuso; plantas sem rizomas; bainhas foliares glabras em toda a extensão

9. P. dichotomiflorum

17. Gluma inferior de ápice truncado; plantas com rizomas; bainhas foliares glabras, pilosas ou hirsutas

26. P. repens

16. Plantas decumbentes

18. Gluma inferior de ápice obtuso; segundo antécio com estípite de $0,2-0,4 \mathrm{~mm}$ compr. 22. $P$. pedersenii

18. Gluma inferior de ápice agudo ou truncado; segundo antécio sem estípite

19. Gluma inferior de ápice truncado ..

26. P. repens

19. Gluma inferior de ápice agudo

20. Plantas com rizomas grossos de entrenós curtos, escamosos; lâminas foliares geralmente involutas, raramente planas, 1-3(-5)mm larg.; bainhas foliares glabras ou pilosas, tricomas subdensos a densos; panícula geralmente subaberta, mais raramente aberta, $1-6 \mathrm{~cm}$ larg.

14. P. gouinii

20. Plantas com rizomas finos de entrenós longos, não escamosos; lâminas foliares planas, 4-12mm larg.; bainhas foliares glabras ou pilosas, tricomas esparsos; panícula aberta a laxa, (4-)6$14(-22,5) \mathrm{cm}$ larg.

3. P. aquaticum

14. Espiguetas com a gluma inferior $1 / 2-4 / 5$ do comprimento da espigueta (exceto em $P$. stramineum em que pode chegar a 1/3); plantas cespitosas, com ou sem rizomas, eretas (às vezes decumbentes em $P$. miliaceum, mas então espiguetas de $(3,7-) 4$ $5,5 \mathrm{~mm}$ compr.), de ambientes abertos, não aquáticos (exceto $P$. aquarum que habitam ambientes aquáticos)

21. Gluma superior caduca na espigueta madura, deixando o dorso do lema superior exposto; segundo antécio negro na maturação

22. Plantas anuais; gluma inferior 3/4-4/5 do comprimento da espigueta 12. P. exiguum

22. Plantas perenes; gluma inferior $1 / 2-3 / 4$ do comprimento da espigueta 
21. Gluma superior persistente na espigueta madura, encobrindo o dorso do lema superior (raramente caducas em algumas espiguetas de $P$. bergii); segundo antécio estramíneo a castanho na maturação

23. Panícula com ramificações inferiores verticiladas, caindo inteira na maturação

23. Panícula com as ramificações inferiores alternas, não caindo inteira na maturação

24. Colmos suculentos, com aerênquima, ramificados nos nós inferiores e superiores; plantas de ambientes aquáticos 2. P. aquarum

24. Colmos não suculentos, sem aerênquima, ramificados apenas nos nós inferiores; plantas de ambientes mesófitos ou úmidos

25. Panícula com os ramos primários adpressos ao eixo principal 8. P. chasei

25. Panícula com os ramos primários divergentes do eixo principal

26. Espiguetas (3,7-)4-5,5mm compr.; panícula (4-)10-26cm compr. 18. P. miliaceum

26. Espiguetas 1,8-3,6(-3,9)mm compr.; panícula 25-45cm compr. (exceto em P. stramineum, que mede 7,5-12,5cm compr., mas então espiguetas de 2,2-2,5mm compr.)

27. Bainhas foliares hirsutas com tricomas urticantes.

28. Plantas com rizomas curtos, até $3 \mathrm{~m}$ de altura; espiguetas 1,8-2,6mm compr.; lâminas foliares 15-35mm larg. 15. P. hirsutum

28. Plantas sem rizomas, $49-110 \mathrm{~cm}$ de altura; espiguetas 3,3-3,6mm compr.; lâminas foliares 8-15mm larg.

19. P. mucronulatum

27. Bainhas foliares glabras ou hirsutas com tricomas não urticantes

29. Espiguetas 3,5-3,6mm compr; bainhas foliares glabras; gluma superior e lema inferior 5(-7)-nervada, 3,1mm compr.; segundo antécio $2,1 \mathrm{~mm}$ compr. ...... 11. P. ephemeroides

29. Espiguetas 2,2-2,5mm compr., bainhas foliares hirsutas; gluma superior e lema inferior 9-11(-13)-nervada, 2,1-2,4mm compr.; segundo antécio 1,6-1,7mm compr.

28. P. stramineum

1. Panicum altum Hitchc. \& Chase, Contr. U.S. Natl. Herb. 17: 488. 1915.

Fig. 1.

México, Caribe, Belize, Guatemala, Honduras, Nicarágua, Panamá, Venezuela e Guiana. Brasil: Região NE (MA).

Material selecionado: BRASIL. Maranhão: Loreto, 20/III/1985, Valls et al. 8433 (CEN, SI).

2. P. aquarum Zuloaga \& Morrone, Novon 1: 185.1991 .

Colômbia e Venezuela. Brasil: N (AM e PA). Material selecionado: BRASIL. Amazonas: Humaitá, 11/V/1980, Gemtchujnicov \& Janssen 369 (IBGE, SP).
3. P. aquaticum Poir. in Lam., Encycl., Suppl., 4: 281. 1816.

Fig. 2.

México, América Central e do Sul. Brasil: N (AC e PA), NE (BA, CE e RN), CO (MS e MT), SE (RJ e SP) e $\mathrm{S}$ (RS e SC).

Material selecionado: BRASIL. Rio Grande do Sul: sem localidade, 1953, Mattos 2293 (BLA).

4. P. bergii Arechav., Anales Mus. Nac. Montevideo 1: 147.1894.

Estados Unidos e América do Sul. Brasil: N (RR), NE (PE), SE (RJ e SP) e S (RS).

Material selecionado: BRASIL. Rio Grande do Sul: Cachoeirinha, 3/II/1999, Guglieri et al. 46 (ICN). 
5. P. campestre Nees ex Trin., Gram. panic. 197. 1826.

Brasil: CO (DF, GO e MT) e SE (MG, RJ e SP). Material selecionado: BRASIL. Goiás: Mineiros, Parque Nacional das Emas, 22/V/1993, Filgueiras 2495 (SI).

6. P. cayennense Lam., Tabl. Encycl. 1: 173. 1791.

México, América Central e do Sul. Brasil: N (PA), NE (MA), CO (MS e MT) e SE (MG).

Material selecionado: BRASIL. Mato Grosso do Sul: Aquidauana, Serra de Maracaju, 12/II/1993, Hatschbach et al. 58947 (SI).

7. P. cervicatum Chase, J. Wash. Acad. Sci 32: 164. 1942.

Fig. 3-4.

Norte da América do Sul até Paraguai. Brasil: N (RO), NE (BA e MA), CO (DF, GO, MS e MT), SE (MG e SP).

Material selecionado: BRASIL. Mato Grosso: Brilhante, Casa Branca, 15/II/1970, Hatschbach 23260 (MBM).

8. P. chasei Roseng., Arr. \& Izag., Bol. Fac. Agr. Montev. 103: 9. 1968.

Fig. 5.

Argentina e Uruguai. Brasil: SE (MG) e S (RS).

Material selecionado: BRASIL. Rio Grande do

Sul: Porto Alegre, Morro Teresópolis, 29/I/1980, Soares 267 (HAS, ICN).

9. P. dichotomiflorum Michx., Fl. bor. amer. 1: 48.1803.

Fig. 6-7.

América do Norte, Central e do Sul. Brasil: N (AM, AP, PA e RR), NE (BA, CE, MA, PE e RN), CO (DF, GO, MS e MT), SE (RJ e SP) e S (PR, RS e SC).

Material selecionado: BRASIL. Rio Grande do Sul: Cachoeira do Sul, X/1933, H.S.A. $60 a$ (ICN).

10. P. elephantipes Nees ex Trin., Gram. panic: 206. 1826.

Fig. 8.

México, América Central e do Sul. Brasil: N (AP, PA e RR), NE (CE), CO (MS e MT) e S (RS).

Material selecionado: BRASIL. Rio Grande do Sul: Guaíba, 13/III/1978, Valls 4004 (ICN).
11. P. ephemeroides Zuloaga \& Morrone, Ann. Missouri Bot. Gard. 83(2): 239. 1996.

Brasil: CO (GO).

Material selecionado: BRASIL. Goiás: Jataí, rodovia BR-364, 3/IV/1986, Valls et al. 9867 (CEN, SI, US).

12. P. exiguum Mez, Bot. Jahrb. Syst. 56, Beibl. 125: 3. 1921.

Colômbia, Peru, Bolívia e Paraguai. Brasil: N (PA e RN), NE (CE, MA, PB, PE e PI), CO (GO, MS e $\mathrm{MT})$ e SE (MG).

Material selecionado: BRASIL. Goiás: Niquelândia, Macedo, 26/VI/1997, Oliveira et al. 766 (IBGE, SI).

13. P. glabripes Döll in Mart., Fl. bras. 2(2): 216. 1877.

Argentina e Uruguai. Brasil: S (PR, RS e SC).

Material selecionado: BRASIL. Paraná: São José dos Pinhais, Roseira, 5/XI/1961, Hatschbach 8444 (MBM).

14. P. gouinii E. Fourn., Mexic. pl. 2: 28. 1886.

Fig. 9.

México até Argentina e Uruguai. Brasil: N (PA), SE (SP) e S (PR e RS).

Material selecionado: BRASIL. Rio Grande do Sul: Eldorado do Sul (Guaíba), Estação Experimental Agronômica da UFRGS, I/1965, Machado s.n. (BLA 4944).

15. P. hirsutum Sw., Fl. Ind. Occ. 1: 173. 1797.

México, América Central e do Sul. Brasil: N (PA). Material selecionado: BRASIL. Pará: Cacanal Grande, s.d., Goeldi 111 (US).

16. P. ligulare Nees ex Trin., Gram. panic.: 206. 1826.

Venezuela. Brasil: NE (BA e PA), CO (DF e GO) e SE (MG).

Material selecionado: BRASIL. Minas Gerais: Joaquim Felício, Serra do Cabral, 14/IV/1996, Hatschbach et al. 64668 (MBM).

17. P. magnispicula Zuloaga, Morrone \& Valls, Iheringia, Bot. 42: 5. 1992.

Brasil: S (SC).

Material examinado: BRASIL. Santa Catarina: 


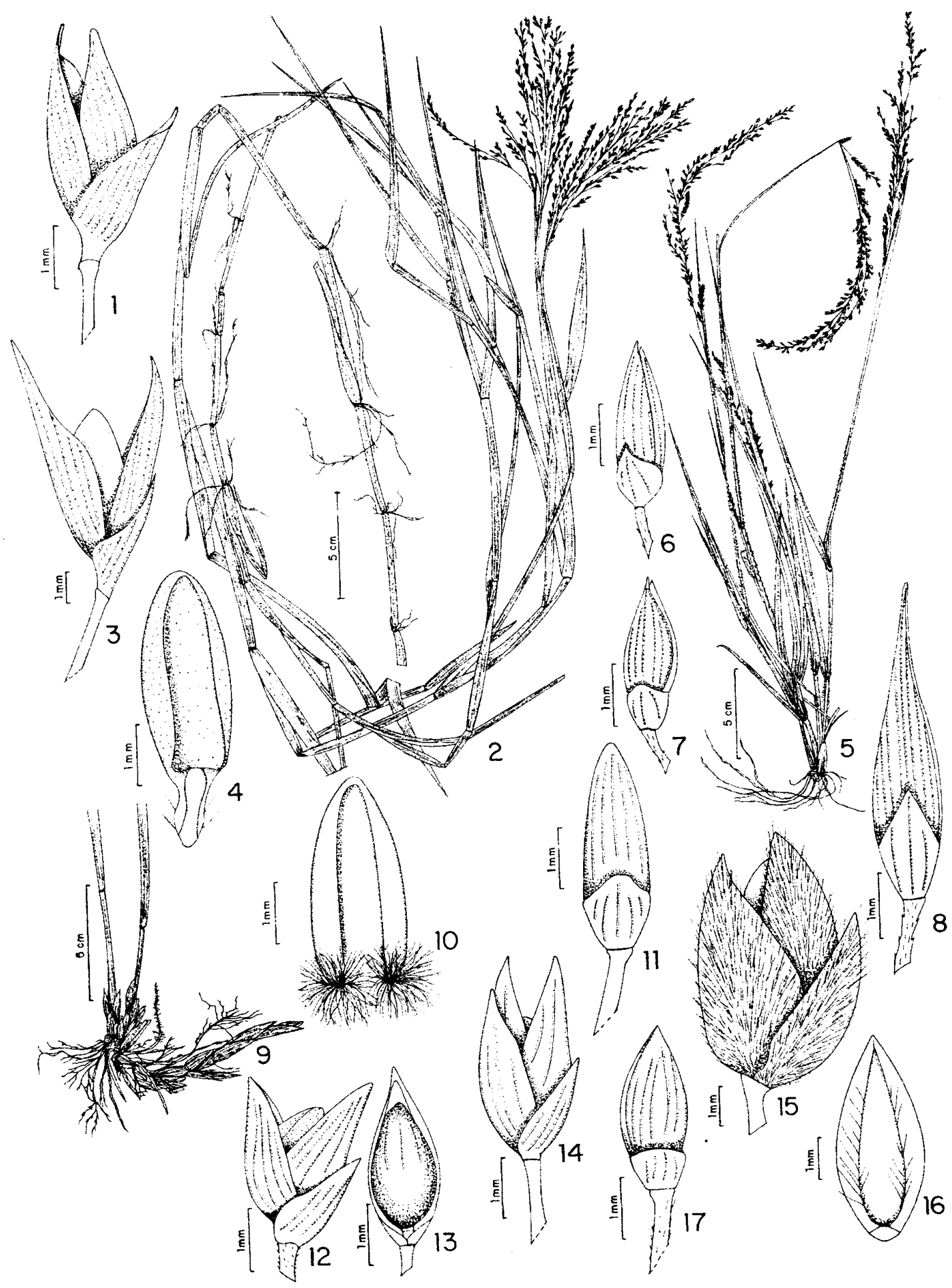

Figuras 1-17. Panicum altum Hitchc. \& Chase. 1. Espigueta com glumas de ápice retorcido (Valls et al. 8433, CEN, SI). P. aquaticum Poir. 2. Hábito (Mattos 2293, BLA). P. cervicatum Chase. 3. Espigueta. 4. Antécio superior, estípite com base pilosa (Hatschbach 23260, MBM). P. chasei Roseng. 5. Hábito, panícula com ramos primários adpressos ao eixo principal (Soares 267, HAS, ICN). P. dichotomiflorum Michx. 6-7. Espiguetas lanceoladas, variação na forma da gluma inferior (H.S.A. 60a, ICN). P. elephantipes Ness ex Trin. 8. Espigueta estreito lanceolada (Valls 4004, ICN). P. gouinii E. Fourn. 9. Rizomas (Machado s.n., BLA 4944). P. olyroides Kunth. 10. Antécio superior com indumento (Longhi-Wagner et al. 1769, BLA, ICN). P. pedersenii Zuloaga. 11. Espigueta, gluma inferior de ápice obtuso (Bueno 1594). P. peladoense Henrard. 12. Espigueta inteira. 13. Espigueta com gluma superior caduca (Pott s.n., BLA 11815). P. quadriglume (Döll) Hitchc. 14. Espiqueta com três antécios (Hatschbach 46144, MBM). P. racemosum (P. Beauv.) Spreng. 15. Espigueta vilosa. 16. Antécio superior com indumento (Winge 219, ICN). P. repens L. 17. Espigueta, gluma inferior de ápice truncado (Black 56-18910, IAN, UB). 
Água Doce, BR 153, 3/XII/1987, Valls et al. 11521 (CEN, SI).

\section{P. miliaceum L., Sp. pl.: 58. 1753.}

Nativa do Velho Mundo, introduzida e cultivada na América, ocorrendo algumas vezes como subespontânea.

Material selecionado: BRASIL. Minas Gerais: Belo Horizonte, 10/V/2000, Macedo 4066 (PAMG).

19. P. mucronulatum Mez, Bot. Jahrb. Syst. 56, Beibl. 125: 2. 1921.

Brasil: NE (AL e BA).

Material selecionado: BRASIL. Bahia: Dom Macedo Costa, 5/VII/1985, Noblick \& Lemos 3955 (MBM, HUEFS).

20. P. mystasipum Zuloaga \& Morrone, Iheringia, Bot. 42: 14. 1992.

Brasil: $\mathrm{CO}(\mathrm{GO})$.

Material selecionado: BRASIL. Goiás: Mineiros, Parque Nacional das Emas, 22/V/1993, Filgueiras 2479 (SI).

21. P. olyroides Kunth, Nov. Gen. Sp. 1: 102. 1816.

Fig. 10.

América do Sul. Brasil: N (PA), NE (MA e PB), $\mathrm{CO},(\mathrm{DF}, \mathrm{GO}, \mathrm{MS}$ e MT), SE (MG e SP) e S (PR, RS e SC).

Material selecionado: BRASIL. Rio Grande do Sul: Porto Alegre, Morro da Polícia, 14/XI/1988, Longhi-Wagner et al. 1769 (BLA, ICN).

22. P. pedersenii Zuloaga, Hickenia 1: 148. 1978.

Fig. 11.

Paraguai e Argentina. Brasil: S (RS).

Material selecionado: BRASIL. Rio Grande do Sul: Porto Alegre, Morro do Côco, 31/VII/1979, Bueno 1594 (ICN).

23. P. peladoense Henrard, Blumea 4: 504. 1941.

Fig. 12-13.

Bolívia, Paraguai, Argentina e Uruguai. Brasil: N (PA), CO (DF, GO, MS e MT), SE (MG e SP) e S (RS e SC).

Material selecionado: BRASIL. Rio Grande do Sul: Eldorado do Sul (Guaíba), Estação Experimental Agronômica da UFRGS, 20/I/1972, Pott s.n.(BLA 11815).
24. P. quadriglume (Döll) Hitchc., Contr. U.S. Natl. Herb. 24: 460. 1927.

Fig. 14.

Peru, Bolívia, Paraguai e Argentina. Brasil: NE (MA), CO (GO, MS e MT) e SE (MG e SP).

Material selecionado: BRASIL. Mato Grosso: Ponta Porã, Ponto Alto, 12/II/1983, Hatschbach 46144 (MBM).

25. P. racemosum (P. Beauv.) Spreng., Syst. veg. 1: 313. 1825.

Fig. 15-16.

Chile, Argentina e Uruguai. Brasil: NE (CE), SE (ES, RJ e SP) e S (RS e SC).

Material selecionado: BRASIL. Rio Grande do Sul: Tramandaí, Nova Tramandaí, 2/XI/1974, Winge 219 (ICN).

26. P. repens L., Sp. pl. 2: 87. 1762.

Fig. 17.

Estados Unidos, Caribe, Belize e Argentina. Brasil: em todo o país, exceto no Rio Grande do Sul.

Material selecionado: BRASIL. Pará: Belém, IAN, 29/XI/1956, Black 56-18910 (IAN, UB).

27. P. rudgei Roem. \& Schult., Syst. veg. 2: 244. 1817.

México, América Central e do Sul. Brasil: N (AM, AP, PA, RO e RR), NE (BA e PE), CO (DF, GO, MS e MT) e SE (MG, RJ e SP).

Material selecionado: BRASIL. São Paulo: Mogi-Guaçu, Fazenda Campininha, 26/I/1996, Longhi-Wagner et al. 3295 (HRCB).

28. P. stramineum Hitchc. \& Chase, Contr. U.S. Natl. Herb 15: 67. 1910.

Estados Unidos, México, Venezuela, Peru, Bolívia, Paraguai e Argentina. Brasil: NE (CE e MA) e CO (MS e MT).

Material selecionado: BRASIL. Mato Grosso do Sul: Miranda, Guaicurus, Fazenda Bodoquena, 25/X/1978, Allem et al. 2145 (CEN, IBGE).

29. P. tricholaenoides Steud., Syn. pl. glumac. 1: 68. 1853.

América do Sul. Brasil: CO (MS), SE (MG) e S (PR, RS e SC).

Material selecionado: BRASIL. Minas Gerais: Formoso, Parque Nacional Grande Sertão Veredas, 
riacho Santa Rita, 5/XII/1997, Alvarenga 1140 (IBGE, SI).

\section{Agradecimentos}

Os autores agradecem aos curadores dos herbários revisados, pelo empréstimo de material. A primeira autora agradece ao $\mathrm{CNPq}$, pela bolsa concedida.

\section{Referências bibliográficas}

Aliscioni, S.S.; Giussani, L.M.; Zuloaga, F.O. \& Kellogg, E.A. 2003. A molecular phylogeny of Panicum (Poaceae: Paniceae). Test of monophyly and phylogenetic placement with the Panicoideae. American Journal of Botany 90(5): 796-821.

Clayton, W.D. \& Renvoize, S.A. 1986. Genera Graminum, Grasses of the world. Her Majesty's Stationery Office, London.

Gould, F.W. 1974. Nomenclatural changes in the Poaceae. Brittonia 29: 59-60.

Guglieri, A. \& Longhi-Wagner, H.M. 2000. Panicum (Gramineae - Paniceae). Flora Ilustrada do Rio Grande do Sul - 26. Boletim do Instituto de Biociências/UFRGS 59: $1-163$.

Häfliger, E. \& Scholz, H. 1980. Panicoid grass weeds. Grass weeds 1. Ciba-Geigy, Switzerland.

Holmgren, P.K.; Holmgren, N.H. \& Barnett, L.C. 1990. Index Herbariorum, 8 ed. New York Botanical Garden. The Herbaria of the world, New York.

Smith, L.B.; Wasshausen, D.C. \& Klein, R.M. 1982. Gramineae. Flora Ilustrada Catarinense (Gram.): 633-756.

Watson, L. \& Dallwitz, M.J. 1992. The grass genera of the world. University Press, Cambridge.

Zuloaga, F.O. 1987a. Systematics of New World species of Panicum (Poaceae: Paniceae). Pp. 287-306. In: T.R. Soderstrom (ed.). Grass Systematics and Evolution. Smithsonian Institution Press, Washington.
Zuloaga, F.O. 1987b. A revision of Panicum subgenus Panicum section Rudgeana (Poaceae: Paniceae). Annals of the Missouri Botanical Garden 74(3): 463-478.

Zuloaga, F.O. 1989. El género Panicum en la República Argentina. III. Darwiniana 29: 289-370.

Zuloaga, F.O. 1994. Panicum. Pp. 211-320. In: F.O. Zuloaga; O. Morrone; Z.E. Rúgolo de Agrasar; A.M. Anton; M.O. Arriaga \& A.M. Cialdella (eds.). Gramineae V. Panicoideae - Paniceae. Flora del Paraguay 23. Missouri Botanical Garden Press, Saint Louis.

Zuloaga, F.O. \& Morrone, O. 1992. Panicum mystasipum (Poaceae: Panicoideae: Paniceae), una nueva especie para el Brasil. Iheringia 42: 13-23.

Zuloaga, F.O. \& Morrone, O. 1996a. Revisión de las especies americanas de Panicum subgénero Panicum sección Panicum (Poaceae: Panicoideae: Paniceae). Annals of the Missouri Botanical Garden 83(2): 200-280.

Zuloaga, F.O. \& Morrone, O. 1996b. New species of Paniceae (Poaceae: Panicoideae) from Brazil. Novon 6(3): 310-317.

Zuloaga, F.O.; Ellis, R.P. \& Morrone, O. 1992a. A revision of Panicum subgenus Phanopyrum section Laxa (Poaceae: Panicoideae: Paniceae). Annals of the Missouri Botanical Garden 79(4): 770-818.

Zuloaga, F.O.; Dubcovsky, J. \& Morrone, O. 1993a. Infrageneric phenetic relations in genus Panicum (Poaceae: Panicoideae: Paniceae): a numerical analysis. Canadian Journal of Botany 71: 1312-1327.

Zuloaga, F.O.; Ellis, R.P. \& Morrone, O. 1993b. A revision of Panicum subg. Dichanthelium sect. Dichanthelium (Poaceae: Panicoideae: Paniceae) in Mesoamerica, the West Indies, and South America. Annals of the Missouri Botanical Garden 80(1): 119-190.

Zuloaga, F.O.; Morrone, O. \& Valls, J.F.M. 1992b. Dos nuevas especies del género Panicum (Poaceae: Paniceae) para el Brasil. Iheringia 42: 3-12.

Zuloaga, F.O.; Morrone, O.; Giussani, L. \& Vega, A. 1998. Revisión y análisis cladístico de Steinchisma (Poaceae: Panicoideae: Paniceae). Annals of the Missouri Botanical Garden 85(4): 631-656.

Zuloaga, F.O.; Guglieri, A. \& Longhi-Wagner, H.M. 2001. Panicum. Pp. 168-190. In: H.M. Longhi-Wagner; V. Bittrich; M.G.L. Wanderley \& G.J. Shepherd (eds.). Poaceae - Flora Fanerogâmica do Estado de São Paulo. v. 1. São Paulo, Hucitec. 
